\title{
Risk Factors Associated with Cardiac Autonomic Modulation in Obese Individuals
}

\author{
Camila Oliveira, ${ }^{1}$ Erika Aparecida Silveira $\left(\mathbb{D},{ }^{2}\right.$ Lorena Rosa, ${ }^{1}$ Annelisa Santos, ${ }^{1}$ \\ Ana Paula Rodrigues $\mathbb{D}^{1},{ }^{1}$ Carolina Mendonça, ${ }^{1}$ Lucas Silva $\mathbb{D}^{1},{ }^{1}$ Paulo Gentil ${ }^{D},{ }^{3}$ \\ and Ana Cristina Rebelo (iD) \\ ${ }^{1}$ Medicine Faculty, Health Science Post-Graduation Program, Universidade Federal de Goiás, Goiânia, Goiás, Brazil \\ ${ }^{2}$ Medicine Faculty, Coordinator of the Severe Obesity Study Group, Federal University of Goiás, Goiânia, Brazil \\ ${ }^{3}$ College of Physical Education and Dance, Federal University of Goiás, Goiânia, Brazil \\ ${ }^{4}$ Department of Morphology, Biological Sciences Institute, Federal University of Goiás, Goiânia, Goiás, Brazil
}

Correspondence should be addressed to Ana Cristina Rebelo; anacristina.silvarebelo@gmail.com

Received 18 January 2020; Accepted 7 March 2020; Published 1 April 2020

Academic Editor: Claire Stocker

Copyright () 2020 Camila Oliveira et al. This is an open access article distributed under the Creative Commons Attribution License, which permits unrestricted use, distribution, and reproduction in any medium, provided the original work is properly cited.

\begin{abstract}
Obesity leads to an imbalance in the autonomic nervous system, especially in increased sympathetic modulation and decreased vagal tone, and some anthropometric, metabolic, and lifestyle variables may increase the risk of developing cardiovascular disease. Objective. To analyze the association between cardiovascular autonomic modulation and biochemical and anthropometric markers, food intake, and physical activity level in severely obese individuals. Methodology. The present study is a cutout of a randomized clinical trial "Effect of nutritional intervention and olive oil in severe obesity" (DieTBra Trial), where the baseline data were analyzed. Anthropometric data, biochemical exams, heart rate variability (HRV), accelerometry, and $24 \mathrm{~h}$ recall (R24H) of obese patients (body mass index BMI $\geq 35 \mathrm{~kg} / \mathrm{m}^{2}$ ) were collected. Results. 64 obese patients were analyzed, with a mean age of $39.10 \pm 7.74$ years (27 to 58 years). By HRV analysis, in the frequency domain, the obese had a higher predominance of sympathetic autonomic modulation (low frequency (LF) $56.44 \pm 20.31 \mathrm{nu}$ ) and lower parasympathetic modulation (high frequency (HF) $42.52 \pm 19.18 \mathrm{nu}$ ). A negative association was observed between the variables Homeostasis Evaluation Model (HOMA-IR) and HF $(p=0.049)$. In the physical activity analysis, there was a negative association between moderate to vigorous physical activity and the sympathetic component $(p=0.043)$, and for sedentary time (ST), there was a negative association with HF $(p=0.049)$ and LF/HF $(p=0.036)$ and a positive association with LF $(p=0.014)$. For multiple linear regression, waist circumference (WC) and HOMA-IR values were negatively associated with $\operatorname{HF}(\beta=-0.685, p=0.010 ; \beta=-14.989, p=0.010$; respectively). HOMA-IR $(\beta=0.141, p=0.003)$ and the percentage of lipids ingested $(\beta=-0.030, p=0.043)$ were negatively associated with LF/HF. Conclusion. Among the cardiovascular risk variables studied, insulin resistance and central adiposity showed the greatest influence on cardiac autonomic modulation of obese, increasing the risk for cardiovascular disease.
\end{abstract}

\section{Introduction}

Studies show that obesity leads to the development of noncommunicable diseases such as hypertension, hypercholesterolemia, insulin resistance, and type 2 diabetes [1-3], and also several previous studies have found changes in cardiac autonomic modulation in overweight and obese individuals [4-8], which may be more affected in obese individuals (body mass index (BMI) $\geq 35.0 \mathrm{~kg} / \mathrm{m}^{2}$ ) [9]. This impairment occurs through an imbalance in the autonomic nervous system (ANS), especially in increased sympathetic modulation and decreased vagal tone leading to decreased heart rate variability (HRV) $[5,7,10]$.

The measurement of these patterns can provide a sensitive and early indicator of serious health impairment in obese individuals with a higher risk of multimorbidity and 
early mortality [9], as they can be indicators of risk of death and cardiovascular disease $[2,11,12]$, such as coronary artery disease, myocardial infarction, sudden death, heart failure and arrhythmias $[7,13]$.

In the literature, there are some studies that show the direct association between sympathetic and/or parasympathetic components and risk factors in obese individuals. Kiviniemi et al. showed an association between reduced parasympathetic modulation and glucose [14]. Vagal modulation was also inversely associated with body fat percentage [15] and with high body mass [16] and waist circumference (WC) $[17,18]$.

Other studies indicate the sympathetic predominance of heart rate in obese, in which there was a negative correlation with Homeostasis Evaluation Model (HOMA-IR) [19], where BMI and peripheral obesity measured by forearm anterior fold demonstrated a positive correlation with low frequency (LF) [20]. Overweight individuals had sympathovagal imbalance due to increased sympathetic activity associated with visceral fat [4]. Physical activity significantly reduced adiposity rates and improved HRV variables related to vagal modulation in sedentary obese individuals [21].

Risk factors are frequently researched, as in the study by Santos et al., cardiometabolic risk factors such as dyslipidemia, HOMA-IR, and hypertension were found in the severely obese patients surveyed, with a significantly higher prevalence in patients with a $\mathrm{BMI} \geq 45 \mathrm{~kg} / \mathrm{m}^{2}$ [22]. However, no study has comprehensively evaluated which HRV indices are associated with cardiovascular risk variables in severely obese subjects, remaining inconclusive among anthropometric (BMI and WC), metabolic (dyslipidemia, hypertension, HOMA-IR, glucose, and insulin), and lifestyle variables (PA, sedentary time (ST), nutritional factors, and eating habits) associated with cardiac autonomic dysfunction.

Therefore, it is essential to determine risk factors for determining cardiac autonomic dysfunction in severely obese individuals, in order to help develop strategies for preventing cardiovascular disease in severely obese individuals. Given this scenario, the objective of the present study was to analyze the association between cardiovascular autonomic modulation and anthropometric, metabolic, and lifestyle variables in severely obese individuals. And the hypothesis of the present study is that the increase in fat concentration may alter the changes in cardiac autonomic modulation and may be associated with other factors of obesity comorbidities.

\section{Materials and Methods}

2.1. Study Design and Ethical Aspects. The present study is a clipping from a randomized clinical trial "Effect of nutritional intervention and olive oil in severe obesity" (DieTBra Trial) (recorded at ClinicalTrials.gov: NCT02463435), where baseline data were analyzed.

Data collection took place at the Clinical Research Unit, Hospital das Clínicas, Federal University of Goiás (UPC/ HC/UFG), Goiânia, Goiás, Brazil, from June 2015 to February 2016. This research was developed according to the recommendations of Resolution No. 466 of December 12, 2012, of the National Health Council (Brazil, 2012) and approved by the UFG HC Research Ethics Committee (CEP/ HC/UFG), under protocol 747.792/2014. All patients who agreed to participate signed the informed consent form.

2.2. Sample Calculation. The sample calculation was estimated from GPower software, version 3.1, based on the mean and standard deviation of HRV indices (HF and LF/ $\mathrm{HF}$ ) obtained in our pilot study. For an alpha of 0.05 and $95 \%$ power, the recommendation was 62 volunteers.

2.3. Target Population and Selection Criteria. Patients with obesity above grade II BMI $\geq 35 \mathrm{~kg} / \mathrm{m}^{2}$ were selected and referred to the Nutrition and Serious Obesity Outpatient Clinic (ANOG/HC/UFG) by the Unified Health System (SUS). Inclusion criteria were patients of both sexes, aged between 18 and 65 years, and $\mathrm{BMI} \geq 35 \mathrm{~kg} / \mathrm{m}^{2}$, calculated based on the following formula: body mass $\div\left[\right.$ height $\left.^{2}\right]$ $\left(\mathrm{kg} / \mathrm{m}^{2}\right)$. We excluded patients after bariatric surgery, patients with weight loss $>8 \%$ in the last trimester, who had received nutritional treatment in the last two years, were using weight loss or anti-inflammatory drugs, patients with HIV/AIDS, heart/kidney/liver failure, obstructive chronic disease, lung disease, and cancer, pregnant women, nursing mothers, and people with physical or mental disabilities. The female volunteers were assessed between the 7 th and 10th days after the start of menstruation, and those who reported using oral contraceptives (with active pills followed by placebo pills) for more than 12 months were assessed during the placebo phase of the medications.

2.4. Data Collection. All volunteers were evaluated at the same time of day to avoid different responses of physiological variables due to circadian changes. Anthropometric data, biochemical examinations, HRV, accelerometry, and $24 \mathrm{~h}$ recall $(\mathrm{R} 24 \mathrm{H})$ were collected.

2.5. Anthropometry. To measure the current body mass, a Welmy digital platform scale with a capacity of $200 \mathrm{~kg}$ and $100 \mathrm{~g}$ accuracy was used. Height was performed on a stadiometer already attached to the digital scale, with an accuracy of $0.1 \mathrm{~cm}$. Body mass and height data were used to calculate BMI, later classified according to WHO (2000) [23].

2.6. Biochemical Tests. Biochemical exams were collected after 12 hours of fasting, in the morning, before the other collection protocols were performed at UPC/UC/UFG and analyzed at Rômulo Rocha Laboratory (HC/UFG). Total cholesterol (TC), high-density lipoprotein (HDL), lowdensity lipoprotein (LDL), very-low-density lipoprotein (VLDL), triglycerides (TG), and Homeostasis Evaluation Model (HOMAR-IR) were analyzed.

2.7. Physical Activity Assessment. The PA level was assessed using the Actigraph model wGT3X accelerometer. The 
equipment was used for 6 consecutive days, 24 hours a day, being affixed to the nondominant wrist. Patients were instructed not to remove the device for sleeping, bathing, or swimming, as the equipment is light, small, and waterproof. WGT3X records acceleration on three axes $(x, y$, and $z)$ at an amplitude of $\pm 8 \mathrm{~g}$ and a frequency of $30 \mathrm{~Hz}$. The data collected were downloaded using ActiLife 6.11.7 software and patients with available data of at least $50 \%$ of the time of use were included in the analysis. The measures used were moderate to vigorous physical activity (MVPA) (>100 mg) lasting at least 10 consecutive minutes per week and sedentary time (ST) $(<50 \mathrm{mg})$ in minutes per day. In the PA level classification, the WHO (2010) recommendation was considered [24] where adults need to perform $\geq 150$ minutes/ week of MVPA.

2.8. 24-Hour Recall. In this research, the food consumption was evaluated by the average of three $\mathrm{R} 24 \mathrm{H}$ in an interval of seven days, being two presential and one via telephone contact. The recall was applied by nutritionists and trainees and consisted of the definition and quantification of all foods consumed the day before. The foods consumed were listed and then asked in detail the size and volume of portions consumed, home measures used, mode of preparation, oil addition, and use of sugars or sweetener [25].

2.9. Heart Rate Variability. For the HRV collection, the volunteers were instructed to remain in the supine resting condition and sitting for an approximate period of 10 minutes, preventing them from moving. Heart rate (HR) was continuously monitored by a heart rate monitor through a chest strap (v800 Polar, Finland). RR interval recordings (iRR) were analyzed from Kubios HRV Analysis software, software version 2.2.

The experiments were carried out in an air-conditioned room, where the ambient temperature was artificially controlled, from an air conditioner, with a temperature between $22^{\circ} \mathrm{C}$ and $24 \underline{\mathrm{o}} \mathrm{C}$ and relative humidity between 50 and $60 \%$. Minimum traffic of people in the environment during the execution of the experiment was maintained.

Each subject was advised not to drink alcohol and/or stimulants 24 hours before and on the day of the tests; not to perform moderate or intense PA on the day before its application; and avoid copious meals and eat a light meal at least two hours before testing.

The iRR variations were used for the linear method analysis in the frequency domain indices, whereby the main HRV evaluation method is made by the spectral analysis that evaluates the autonomic function. The components analyzed were $\mathrm{HF}$ (high frequency), ranging from 0.15 to $0.4 \mathrm{~Hz}$, and LF (low frequency), ranging from 0.04 to $0.15 \mathrm{~Hz}$. LF is considered an index related to sympathetic and vagal modulation in the sinus node, whereas HF is the index related to vagus nerve activity over the heart, and the LF/HF ratio is used as an indirect index of sympathovagal balance $[26,27]$. Measurements of these components were expressed in normalized units (nu).
2.10. Statistical Methods. First, a descriptive analysis was performed: absolute and relative frequency, mean, and standard deviation. The Kolmogorov-Smirnov test was applied to verify the normality of continuous variables and the Levene test was used to verify the homogeneity of the variables. All variables were considered normal and/or homogeneous. Simple linear regression was performed to identify the association between cardiovascular autonomic modulation variables (HF (nu), LF (nu), and LF/HF) and BMI $\left(\mathrm{kg} / \mathrm{m}^{2}\right)$, WC $(\mathrm{cm})$, HOMA-IR ( $\left.\mathrm{mg} / \mathrm{dl}\right)$, insulin ( $\mathrm{um}$ ), blood glucose (mg/dl), MVPA (min/week), ST (min/day), total energy value (TEV) (kcal/day), macronutrients (\%), and SBP and DBP (mmHg). Variables with $p<0.20$ in the simple linear regression were entered in the multiple linear regression (MLR). MLR was performed between the frequency domain indices and the adjusted variables $\mathrm{WC}(\mathrm{cm})$, HOMA-IR (mg/dL), insulin (ui), blood glucose ( $\mathrm{mg} / \mathrm{dL}$ ), MVPA (min/week), TS (min/day), VET (kcal/day), carbohydrates (\%), and lipids (\%). Statistical analysis was performed in the program Statistical Package for the SocialSciences (SPSS; IBM Corp., NY, USA), version 20.0. Statistical significance was previously established at $p<0.05$.

\section{Results}

Of the 64 obese patients analyzed in the present study, 9 were male (14.06\%) and 55 female (85.93\%), as shown in Figure 1, with a mean age of $39.10 \pm 7.74$ years (27 to 58 years). Regarding food intake, for the TEV, an average of $1956.76 \pm 1097.21 \mathrm{kcal} /$ day was obtained and the amount of macronutrients consumed as a percentage for carbohydrates $(53.39 \pm 10.78)$, proteins $(17.90 \pm 6.09)$, and lipids $(28.1 \pm 8.70)$.

The biochemical results of blood are shown in Table 1 . For the anthropometric data evaluated, a mean BMI of $46.61 \pm 6.86 \mathrm{~kg} / \mathrm{m}^{2}$ was observed, with a more frequent obesity degree of 40 to $49.9 \mathrm{~kg} / \mathrm{m}^{2}$ (60.93\%). The mean WC was $118.83 \pm 10.66$, with the highest risk for all men and $84.37 \%$ of women. Patients were shown to be insulin-resistant (HOMA-IR $6.03 \pm 4.10 \mathrm{mg} / \mathrm{dl}$ ). The amount of minutes spent per day on ST and MVPA in minutes per week is also shown in Table 1.

The HRV analysis results from the frequency domain linear analysis showed that the severely obese had a higher predominance of sympathetic autonomic modulation (LF $56.44 \pm 20.31 \mathrm{nu})$ and lower parasympathetic modulation (HF $42.52 \pm 19.18 \mathrm{nu}$ ) in Figure2)we can see an example of comparison between the data of two individuals, one obese and the other non-obese, from the analysis in the frequency domain.

From the simple linear regression analysis, the BMI, WC, TEV, carbohydrate (\%), lipid (\%), protein (\%),SBP, and DBP were not associated with cardiovascular autonomic modulation $(p>0.05)$ (Table 2). However, a negative association was observed between the variables HOMA-IR and HF $(p=0.049)$, and HOMA-IR and LF/HF $(p \leq 0.001)$. For insulin and glycemia, there was a negative association with sympathovagal balance $(p=0.002$ and $p=0.021$, respectively). In the analysis of PA, there was a negative association 


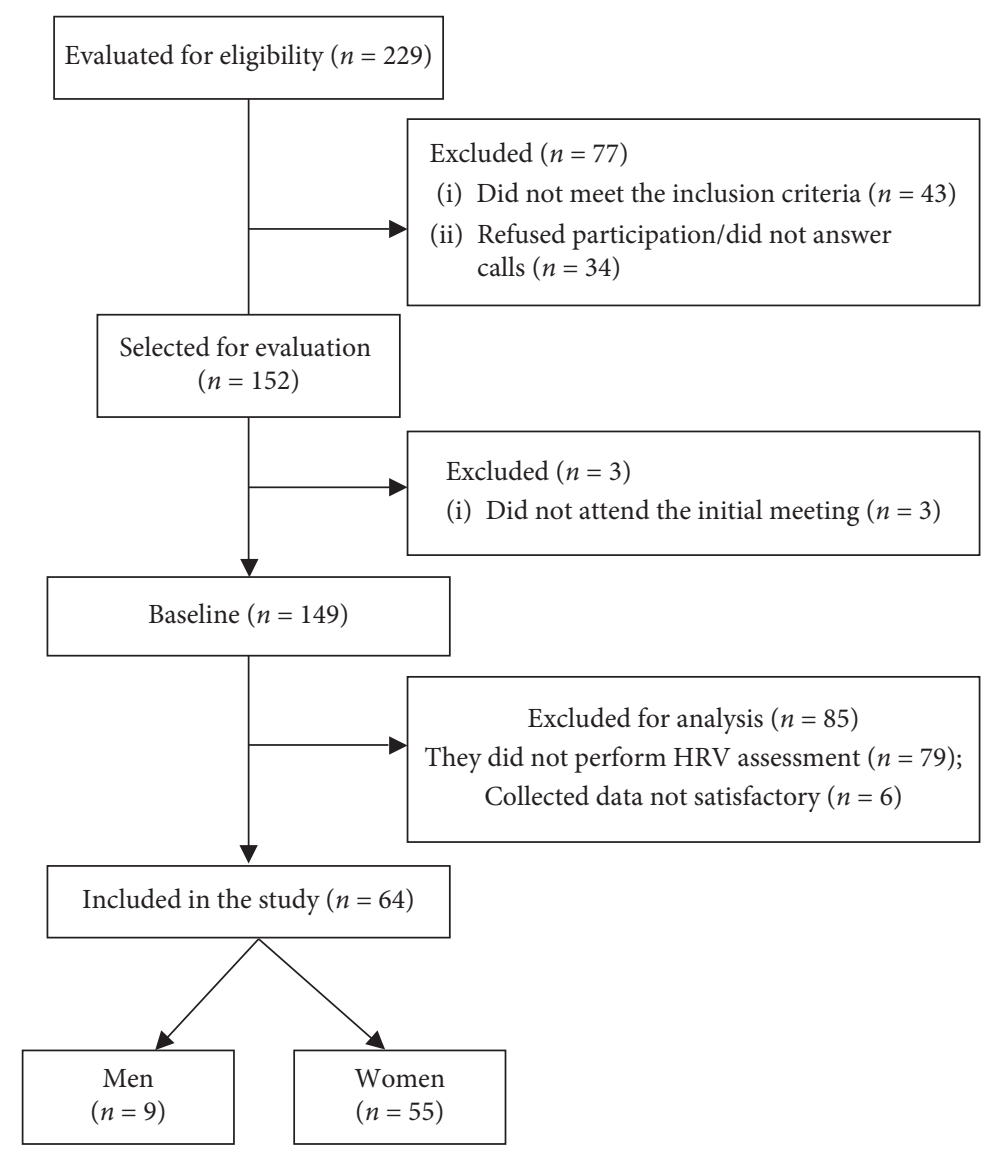

FIGURE 1: Diagram of the flow of selection and continuity of study participants.

between MVPA and the sympathetic component $(p=0.042)$, and for the ST, there was a negative association with HF $(p=0.049)$ and LF/HF $(p=0.036)$ and a positive association with $\operatorname{LF}(p=0.014)$.

In Table 3, from the multiple linear regression, the $\mathrm{WC}$ and HOMA-IR values were negatively and significantly associated with HF $(p=0.010)$. HOMA-IR and percentage lipid values were negatively associated with LF/HF ( $p=0.003$ and $p=0.043$, respectively). No associations were observed between insulin, glycemia, MVPA, ST, TEV, and carbohydrate (\%) with autonomic modulation.

\section{Discussion}

Among the cardiovascular risk factors analyzed in the present study, it was found that insulin resistance and central adiposity were independently associated with changes in HRV parameters in the frequency domain (LF, HF, and LF/ $\mathrm{HF}$ ) and that these altered factors in the population in question have a strong relationship with the dysregulation in cardiac autonomic function and may be the mechanism for the increased prevalence of cardiovascular diseases in obesity.

In the present study, it was observed a negative association between HOMA-IR and parasympathetic modulation and a positive association with global variability was observed. A similar association was reported in a study from our research group in which participants with metabolic syndrome had elevated blood glucose levels associated with reduced parasympathetic modulation [14]. Also in this perspective, insulin was inversely associated with HRV among patients with higher levels of obesity and lower levels of PA [28], since the severely obese in the present study showed a low amount of minutes spent on MVPA (98.92 $\pm 41.00 \mathrm{~min} /$ week).

Our sample showed high body mass and accumulation of visceral fat (BMI $46.61 \pm 6.86 \mathrm{~kg} / \mathrm{m}^{2}$ and $\mathrm{WC}$ $118.83 \pm 10.66 \mathrm{~cm})$, and in this sense, adiposity and especially central fat can lead to insulin resistance, which may alter the function of the autonomic nervous system. Another important point found in the present study was WC associated with worsening vagal tone, in which central obesity, rather than general fat, is related to the impairment of autonomic nervous system function [20]. Corroborating our data, the study by Yadav et al. observed an increase in waistto-hip ratio strongly associated with reduced cardiac parasympathetic activity in obese individuals, and there may be a higher risk of cardiovascular morbidity and mortality due to cardiac autonomic changes [8].

Regarding food consumption, a negative association was observed between the percentage of lipids consumed and the global variability, implying that lipids exert a protective factor in cardiac autonomic function. The fatty acid profile found in dietary fat has different consequences on the 
TABle 1: Clinical characteristics of severely obese adults, Goiânia, Goiás.

\begin{tabular}{|c|c|c|}
\hline Variables & $n(\%)$ & Mean \pm SD \\
\hline Age (years) & & $39.10 \pm 7.74$ \\
\hline $18-29$ & $7(10.93)$ & \\
\hline $30-39$ & $26(40.62)$ & \\
\hline $40-49$ & $23(35.93)$ & \\
\hline$\geq 50$ & $8(12.5)$ & \\
\hline BMI $\left(\mathrm{kg} / \mathrm{m}^{2}\right)$ & & $46.61 \pm 6.86$ \\
\hline $35.00-39.9$ & $9(14.06)$ & \\
\hline $40.00-49.9$ & $39(60.93)$ & \\
\hline$>50.00$ & $16(25)$ & \\
\hline \multicolumn{3}{|l|}{ Dyslipidemia } \\
\hline Total cholesterol <190 (mg/dl) & & $192.72 \pm 41.14$ \\
\hline Yes & $35(54.68)$ & \\
\hline No & $29(45.31)$ & \\
\hline HDL-c >40 (mg/dl) & & $50.10 \pm 12.89$ \\
\hline Yes & $56(87.5)$ & \\
\hline No & $8(12.5)$ & \\
\hline Triglycerides < 150 (mg/dl) & & $158.92 \pm 67.00$ \\
\hline Yes & $30(46.87)$ & \\
\hline No & $34(53.12)$ & \\
\hline LDL-c <70 (mg/dl) & & $110.26 \pm 38.68$ \\
\hline Yes & $4(6.25)$ & \\
\hline No & $60(93.75)$ & \\
\hline HOMA-IR (mg/dl) & & $6.03 \pm 4.10$ \\
\hline Normal & $12(18.75)$ & \\
\hline Changed & $52(81.25)$ & \\
\hline Blood glucose (mg/dl) & & $104.69 \pm 35.52$ \\
\hline$\leq 99$ & $41(64.06)$ & \\
\hline $100-125$ & $12(18.75)$ & \\
\hline$\geq 126$ & $11(17.18)$ & \\
\hline MVPA, $\geq 150$ (min/week) & & $98.92 \pm 41.00$ \\
\hline Yes & $4(6.25)$ & \\
\hline No & $60(93.75)$ & \\
\hline Sedentary time (min/day) & & $1180.55 \pm 81.51$ \\
\hline Normal $<1000$ & $1(1.65)$ & \\
\hline Changed $\geq 1000$ & $63(98.43)$ & \\
\hline \multicolumn{3}{|l|}{ Hypertension $\leq 120 / 80(\mathrm{mmHg})$} \\
\hline SBP & & $126.66 \pm 17.02$ \\
\hline DBP & & $84.33 \pm 10.05$ \\
\hline Yes & $29(45.31)$ & \\
\hline No & $35(54.68)$ & \\
\hline \multicolumn{3}{|l|}{ Diabetes mellitus 2} \\
\hline Yes & $18(28.12)$ & \\
\hline No & $46(71.87)$ & \\
\hline
\end{tabular}

Values expressed as mean \pm standard deviation. BMI = body mass index; $\mathrm{WC}=$ waist circumference; HDL-c $=$ high-density lipoprotein cholesterol; LDL- $\mathrm{c}=$ low-density lipoprotein; VLDL-c = very-low-density lipoprotein; HOMAR-IR = homeostasis evaluation model; MVPA: moderate to vigorous physical activity; $\mathrm{SBP}=$ systolic blood pressure; $\mathrm{DBP}=$ diastolic blood pressure.

human body [29]. It is well established in the literature that excessive saturated fatty acid consumption is associated with increased risk of cardiovascular disease and may reduce the risk of sudden death in cardiovascular disease patients [30], whereas ingestion of unsaturated fatty acids as monounsaturated and especially polyunsaturated fatty acids (PUFAs) has been associated with reduced cardiac mortality [31], with beneficial effect on blood lipid profile in patients with coronary artery disease, and may also be associated with a reduction in the frequency of ventricular tachyarrhythmias in implantable defibrillator cardioverter (ICD) recipients with ischemic cardiomyopathy [32], and in animal models, prevent fatal ischemia-induced cardiac arrhythmias [33] [30].

Also in a review study, La Rovere and Christensen identified that PUFAs can have positive health effects on cardiovascular control in humans, especially on parasympathetic function. One explanation would be that these fatty acids affect the expression of genes involved in inflammation and lipid metabolism [34]. For the present study, we assume that the protective effect that lipid ingestion promoted on the overall cardiovascular variability was perhaps due to the consumption of unsaturated fatty acids, since these exert a reduction in cardiovascular events.

Regarding PA, it was observed that higher levels of MVPA were associated with reduced LF; in this sense, higher levels of PA in severely obese induce a positive response of cardiac sympathetic modulation, and PA may be a cardiac protector, whereas increased sympathetic tone results in increases in heart rate and blood pressure [35], which may increase the risk of cardiovascular disease occurrences.

So being physically active reduces the risks for obesityrelated cardiovascular disease by positively influencing autonomic nerve function $[2,16,36]$ and insulin resistance [37], besides reducing the action of catecholamines (attenuating acetylcholine-oriented vasoconstriction), which reduce resting $\mathrm{HR}$, and during submaximal exercise, allowing for faster HR recovery, which indicates improved vagal modulation and less risk for cardiovascular outcome $[38,39]$. In this scenario, healthy lifestyle was associated with higher HRV, suggesting a positive effect on ANS and throughout life. PA was positively and independently related to HRV in obese adults, implying that regular physical activity induces structural and functional adaptations in the cardiovascular system $[16,40]$.

In the present study, for ST there was a correlation with all the frequency domain indices, in which it correlated negatively with parasympathetic component and the sympathovagal HRV balance and positively with cardiac sympathetic modulation. In line with this, the study by Lião et al. showed a higher cardiac frequency and a decrease in the HF index in overweight elderly people and a lower HRV in the group with low physical capacity compared to the group with high physical capacity [41], implicating that ST is related to increased risk of developing cardiovascular disease, in which the occurrence of a heart attack is twice as high in sedentary individuals when compared to those who are regularly active [42], all-cause mortality, and reduced life expectancy [43].

Among the limitations of our study, not having a control group for comparison with the population in question was an important factor. Nevertheless, the data presented indicate the importance to modify the lifestyle in severely obese by performing PA.

Control of risk factors in obesity is extremely important as they produce significant effects on the cardiovascular autonomic system and may be fundamental to the health of these patients. Anthropometric assessment and identification of risk factors alone or in combination can be useful for 


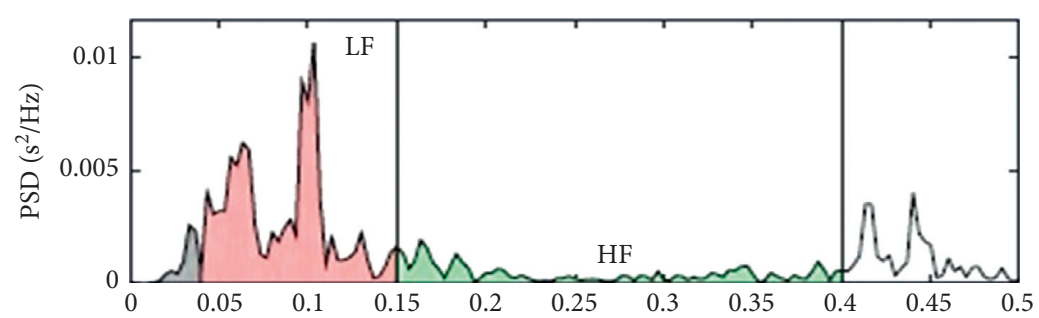

(a)

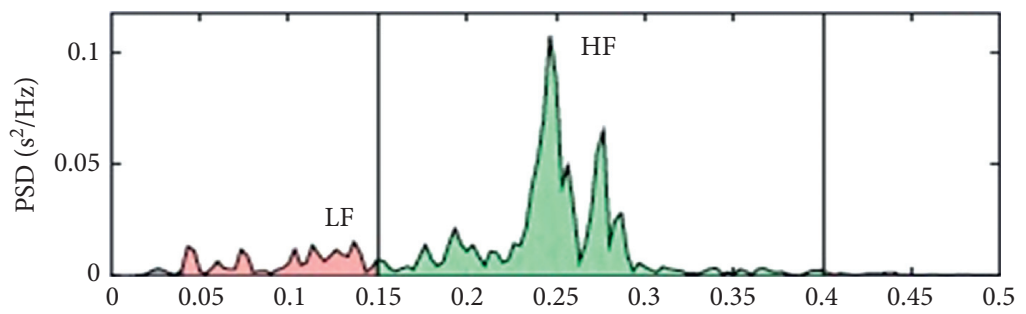

(b)

FIGURE 2: Graph in normalized unit with LF and HF values comparing (a) a severely obese woman: 34 years, weight $94 \mathrm{~kg}$, height $1.62 \mathrm{~m}$, and body mass index $35.8 \mathrm{~kg} / \mathrm{m}^{2}$, and (b) and a nonobese woman: 35 years, weight $59.4 \mathrm{~kg}$, height $1.59 \mathrm{~m}$, and body mass index $23.6 \mathrm{~kg} / \mathrm{m}^{2}$; $\mathrm{HF}=$ high frequency; $\mathrm{LF}=$ low frequency.

TABLE 2: Linear regression among cardiovascular autonomic modulation variables with BMI, WC, HOMA-IR, insulin, blood glucose, MVPA, ST, TEV, percentage macronutrients, SBP, and DBP.

\begin{tabular}{lccccccccc}
\hline & \multicolumn{3}{c}{ HF $(\mathrm{nu})$} & & \multicolumn{3}{c}{ LF $(\mathrm{nu})$} & \multicolumn{3}{c}{ LF/HF } \\
Variables & $\beta$ & $95 \% \mathrm{CI}$ & $p$ value & $\beta$ & $95 \%$ CI & $p$ value & $\beta$ & $95 \%$ CI & $p$ value \\
\hline BMI (kg/m ${ }^{2}$ ) & 2.756 & $-3.646-9.157$ & 0.393 & 0.991 & $-7.286-9.268$ & 0.812 & -0.008 & $-0.064-0.049$ & 0.792 \\
WC (cm) & 1.252 & $-2.877-5.382$ & 0.547 & 4.735 & $-0.453-9.924$ & 0.073 & -0.014 & $-0.050-0.022$ & 0.439 \\
HOMA-IR (mg/dl) & -11.540 & $-23.042--0.040$ & $0.049^{*}$ & 13.726 & $-1.133-28.584$ & 0.070 & -0.168 & $-0.253--0.084$ & $<0.001^{*}$ \\
Insulin (ui) & -3.270 & $-7.005-0.464$ & 0.085 & 3.416 & $-1.426-8.259$ & 0.163 & -0.048 & $-0.078--0.019$ & $0.002^{*}$ \\
Blood glucose (mg/dl) & -0.762 & $-1.999-0.474$ & 0.222 & 0.941 & $-0.651-2.532$ & 0.242 & -0.012 & $-0.023--0.002$ & $0.021^{*}$ \\
MVPA (min/day) & -0.844 & $-1.898-0.210$ & 0.114 & -1.389 & $-2.726-0.051$ & $0.042^{*}$ & 0.005 & $-0.005-0.014$ & 0.303 \\
ST (min/day) & -0.528 & $0.002-1.053$ & $0.049^{*}$ & 0.837 & $0.173-1.502$ & $0.014^{*}$ & -0.005 & $-0.010--0.000$ & $0.036^{*}$ \\
TEV (kcal/day) & 0.021 & $-0.019-0.061$ & 0.300 & 0.041 & $-0.010-0.092$ & 0.110 & -0.000 & $-0.001-0.000$ & 0.146 \\
Carbohydrate (\%) & 0.837 & $-4.914-3.241$ & 0.683 & -2.686 & $-7.892-2.521$ & 0.306 & 0.028 & $-0.007-0.063$ & 0.118 \\
Lipid (\%) & 2.689 & $-2.322-7.700$ & 0.288 & 5.404 & $-0.953-11.762$ & 0.094 & 0.037 & $-0.081-0.006$ & 0.091 \\
Protein (\%) & -2.868 & $-10.056-4.321$ & 0.428 & -2.619 & $-11.886-6.648$ & 0.574 & -0.012 & $-0.075-0.052$ & 0.720 \\
SBP (mmHg) & -41.830 & $-0.2344-0.186$ & 0.822 & 42.47 & $-0.186-0.233$ & 0.823 & 3.480 & $-2.305-2.870$ & 0.825 \\
DBP (mmHg) & -144.600 & $-0.141-0.122$ & 0.912 & 158.9 & $-0.127-0.140$ & 0.925 & 8.708 & $-1.540-1.769$ & 0.890 \\
\hline
\end{tabular}

$\mathrm{HF}=$ high frequency; $\mathrm{LF}=$ low frequency; $\mathrm{LF} / \mathrm{HF}=$ low frequency/high frequency ratio; nu: normalized units; $\mathrm{BMI}=$ body mass index; $\mathrm{WC}=$ waist circumference; HOMAR-IR = Homeostasis Evaluation Model; MVPA = average of minutes per day spent in vigorous physical activity $(>100 \mathrm{mg}) ; \mathrm{ST}=$ sedentary time; $\mathrm{VET}=$ total energy value $(\mathrm{kcal} / \mathrm{day}), \mathrm{SBP}=$ systolic blood pressure, $\mathrm{DBP}=$ diastolic blood pressure. ${ }^{*} p<0.05$.

TABle 3: Multiple linear regression between frequency domain indices and adjusted variables WC, HOMA-IR, insulin, blood glucose, MVPA, ST, TEV, carbohydrate, and lipid.

\begin{tabular}{lccccccccc}
\hline & \multicolumn{3}{c}{ HF (nu) } & \multicolumn{3}{c}{ LF (nu) } & \multicolumn{3}{c}{ LF/HF } \\
Variables & Adjusted $\beta$ & 95\% CI & $p$ value & Adjusted $\beta$ & $95 \%$ CI & $\begin{array}{c}p \\
\text { value }\end{array}$ & Adjusted $\beta$ & $95 \%$ CI & $p$ value \\
\hline WC (cm) & -0.685 & $0.169-1.201$ & $0.010^{*}$ & 2.792 & $-2.984-8.569$ & 0.337 & -0.002 & $-0.007-0.002$ & 0.282 \\
HOMA-IR (mg/dl) & -14.989 & $-26.273--3.705$ & $0.010^{*}$ & 8.670 & $-6.480-23.819$ & 0.257 & 0.141 & $-0.231--0.051$ & $0.003^{*}$ \\
Insulin (ui) & -0.038 & & 0.924 & 0.237 & & 0.589 & -0.206 & 0.647 \\
Blood glucose (mg/dl) & - & & - & - & & - & -0.205 & - \\
MVPA (min/day) & -0.196 & & 0.381 & 0.181 & & 0.447 & - & - \\
ST (min/day) & -0.318 & & 0.169 & 0.687 & $-0.015-1.390$ & 0.055 & -0.459 & - \\
TEV (kcal/day) & 0.007 & & 0.954 & 0.034 & $-0.017-0.858$ & 0.183 & - & -0.085 \\
Carbohydrate (\%) & - & - & - & - & - & - & -0.008 & $-0.068-0.053$ & 0.803 \\
Lipid (\%) & - & & 0.699 & 3.543 & $-2.832-9.917$ & 0.270 & -0.030 & $-0.106-0.046$ & $0.043^{*}$ \\
\hline
\end{tabular}

$\mathrm{LF}=$ low frequency; $\mathrm{HF}=$ high frequency; $\mathrm{LF} / \mathrm{HF}=$ low frequency/high frequency ratio, nu: normalized units; WC= waist circumference; HOMARIR = Homeostasis Evaluation Model; MVPA = average of minutes per day spent in vigorous physical activity $(>100 \mathrm{mg}) ; \mathrm{ST}=$ sedentary time; TEV=total energy value (kcal/day). ${ }^{*} p<0.05$. 
planning and implementing public policies aimed at reducing morbidity and mortality from cardiovascular disease [42]. Therefore, the information in this study is extremely relevant, since it investigated the cardiovascular risk factors that affect severely obese individuals and observed that the main factors that affect the alteration of autonomic function are insulin resistance, central adiposity, MPVA, and ST.

This demonstrates the importance of adopting strategies to change the lifestyle of the severely obese [44], mainly due to the practice of physical activity as a nonmedication strategy and primary prevention for the treatment of obesity [45], along with additional weight gain with the maintenance of weight loss of those who reach it [46]. Public health programs should be promoted with a multidisciplinary approach in which health professional can better advise the severely obese, physically, foodily and psychologically. Multidisciplinary public health policy programs should be promoted, where health professionals can better advise the severely obese, physically, foodily, and psychologically.

\section{Conclusion}

The study reveals that among cardiovascular risk factors, insulin resistance and central adiposity were independently associated with cardiac autonomic modulation in obese individuals, having fundamental importance in altering cardiac autonomic modulation of obesity.

\section{Data Availability}

Data used to support the results of this study are available from the corresponding author upon request.

\section{Conflicts of Interest}

The authors declare no conflicts of interest.

\section{Acknowledgments}

The authors thank all the patients who participated in the study and the collaborators who were part of this project and also thank the Clinical Research Unit of the Hospital das Clinicas of the Federal University of Goiás. This study was partially funded by the Goiás State Research Support Foundation (FAPEG) (grant no. 201310267000003).

\section{References}

[1] J. Upadhyay, O. Farr, N. Perakakis, W. Ghaly, and C. Mantzoros, "Obesity as a disease," Medical Clinics of North America, vol. 102, no. 1, pp. 13-33, 2018.

[2] C. Voulgari, S. Pagoni, A. Vinik, and P. Poirier, "Exercise improves cardiac autonomic function in obesity and diabetes," Metabolism, vol. 62, no. 5, pp. 609-621, 2013.

[3] M. Chrostowska, A. Szyndler, M. Hoffmann, and K. Narkiewicz, "Impact of obesity on cardiovascular health," Best Practice \& Research Clinical Endocrinology \& Metabolism, vol. 27, no. 2, pp. 147-156, 2013.

[4] K. K. Chintala, B. H. Krishna, and N. Mallikarjuna Reddy, "Heart rate variability in overweight health care students: correlation with visceral fat," Journal of Clinical and Diagnostic Researc, vol. 9, no. 1, pp. CC06-CC08, 2015.

[5] R. C. Rossi, L. C. M. Vanderlei, A. C. C. R. Gonçalves et al., "Impact of obesity on autonomic modulation, heart rate and blood pressure in obese young people," Autonomic Neuroscience, vol. 193, pp. 138-141, 2015.

[6] R. D. Raimundo and J. J. Godleski, "Heart rate variability in metabolic syndrome," Journal of Human Growth and Development, vol. 25, no. 1, pp. 8-12, 2015.

[7] A. Malliani and N. Montano, "Current perspective heart rate variability as a clinical tool," Italian Heart Journal, vol. 3, no. 8, pp. 439-445, 2002.

[8] R. L. Yadav, P. K. Yadav, L. K. Yadav, K. Agrawal, S. K. Sah, and M. N. Islam, "Association between obesity and heart rate variability indices: an intuition toward cardiac autonomic alteration-a risk of CVD," Diabetes, Metabolic Syndrome and Obesity: Targets and Therapy, vol. 10, pp. 57-64, 2017.

[9] M. de Sant Anna Junior, J. R. I. Carneiro, R. F. Carvalhal et al., "Cardiovascular autonomic dysfunction in patients with morbid obesity," Arquivos Brasileiros de Cardiologia, vol. 105, no. 6, pp. 580-587, 2015.

[10] J. Indumathy, G. K. Pal, P. Pal et al., "Association of sympathovagal imbalance with obesity indices, and abnormal metabolic biomarkers and cardiovascular," Obesity Research \& Clinical Practice, vol. 9, no. 1, pp. 55-66, 2014.

[11] B. Xhyheri, O. Manfrini, M. Mazzolini, C. Pizzi, and R. Bugiardini, "Heart rate variability today," Progress in Cardiovascular Diseases, vol. 55, no. 3, pp. 321-331, 2012.

[12] V. C. Kunz, R. B. Souza, A. C. M. Takahashi, A. M. Catai, and E. Silva, "The relationship between cardiac autonomic function and clinical and angiographic characteristics in patients with coronary artery disease," Brazilian Journal of Physical Therapy, vol. 15, no. 6, pp. 503-510, 2011.

[13] H. K. Jamali, F. Waqar, and M. C. Gerson, "Cardiac autonomic innervation," Journal of Nuclear Cardiology, vol. 24, no. 5, pp. 1558-1570, 2017.

[14] L. R. B. E. Silva, A. R. Zamunér, P. Gentil et al., "Cardiac autonomic modulation and the kinetics of heart rate responses in the on- and off-transient during exercise in women with metabolic syndrome," Frontiers in Physiology, vol. 8, pp. 1-9, 2017.

[15] A. M. Kiviniemi, N. Perkiömäki, J. Auvinen et al., "Fitness, fatness, physical activity, and autonomic function in midlife," Medicine \& Science in Sports \& Exercise, vol. 49, no. 12, pp. 2459-2468, 2017.

[16] K. M. Kaikkonen, R. i. Korpelainen, M. P. Tulppo et al., "Physical activity and aerobic fitness are positively associated with heart rate variability in obese adults," Journal of Physical Activity and Health, vol. 11, no. 8, pp. 1614-1621, 2014.

[17] R. Franz, M. A. Maturana, J. A. Magalhães, R. S. Moraes, and P. M. Spritzer, "Central adiposity and decreased heart rate variability in postmenopause: a cross-sectional study," Climacteric, vol. 16, no. 5, pp. 576-583, 2013.

[18] J. Koenig, B. G. Windham, L. Ferrucci et al., "Association strength of three adiposity measures with autonomic nervous system function in apparently healthy employees," The Journal of Nutrition, Health and Aging, vol. 19, no. 9, pp. 879-882, 2014.

[19] A. Espinoza-Salinas, E. Zafra-Santos, G. Pavez-Von Martens, C. Cofré-Bolados, J. Lemus-Zúñiga, and P. Sánchez-Aguilera, "Análisis de variabilidad del ritmo cardiaco y su relación con la sensibilidad insulínica en pacientes obesos y con sobrepeso," Revista médica de Chile, vol. 143, no. 9, pp. 1129-1135, 2015. 
[20] M. Rastović, B. Srdić-Galić, O. Barak, and E. Stokić, "Association between anthropometric measures of regional fat mass and heart rate variability in obese women," Nutrition \& Dietetics, vol. 74, no. 1, pp. 51-60, 2017.

[21] J. Phoemsapthawee, P. Prasertsri, and N. Leelayuwat, "Heart rate variability responses to a combined exercise training program: correlation with adiposity and cardiorespiratory fitness changes in obese young men," Journal of Exercise Rehabilitation, vol. 15, no. 1, pp. 114-122, 2019.

[22] A. S. A. C. Santos, A. P. S. Rodrigues, L. P. S. Rosa, and E. A. Silveira, "Cardiometabolic risk factors and Framingham Risk Score in severely obese patients: baseline data from DieTBra Trial," Nutrition, Metabolism and Cardiovascular Diseases, vol. 30, no. 3, pp. 474-482, 2020.

[23] World Health Organization, Obesity: Preventing and Managing the Global Epidemic, World Health Organization, Geneva, Switzerland, 2000.

[24] World Health Organization, Global Recommendations on Physical Activity for Health, World Health Organization, Geneva, Switzerland, 2010.

[25] R. Pereira and R. A. Sichieri, Epidemiologia Nutricional, 1a Edição, Rio de Janeiro, Brazil, 2007.

[26] Task Force of the European Society Electrophysiology, "Heart rate variability," Circulation, vol. 93, no. 5, pp. 1043-1065, 1996.

[27] C. R. Bellenger, R. L. Thomson, E. Y. Robertson et al., “The effect of functional overreaching on parameters of autonomic heart rate regulation," European Journal of Applied Physiology, vol. 117, no. 3, pp. 541-550, 2017.

[28] L. E. Charles, M. E. Andrew, K. Sarkisian et al., "Associations between insulin and heart rate variability in police officers," American Journal of Human Biology, vol. 26, no. 1, pp. 56-63, 2014.

[29] M. Haag and N. G. Dippenaar, "Dietary fats, fatty acids and insulin resistance: short review of a multifaceted connection," Medical Science Monitor, vol. 11, no. 12, pp. RA359-67, 2005.

[30] S. O. E. Ebbesson, V. S. Voruganti, P. B. Higgins et al., "Fatty acids linked to cardiovascular mortality are associated with risk factors," International Journal of Circumpolar Health, vol. 74, no. 1, Article ID 28055, 2015.

[31] P. L. Mclennan, "Cardiac physiology and clinical efficacy of dietary fish oil clarified through cellular mechanisms of omega-3 polyunsaturated fatty acids," European Journal of Applied Physiologyl, vol. 114, no. 7, pp. 1333-1356, 2014.

[32] D. Weisman, R. Beinart, A. Erez et al., "Effect of supplemented intake of omega-3 fatty acids on arrhythmias in patients with ICD: fish oil therapy may reduce ventricular arrhythmia," Journal of Interventional Cardiac Electrophysiology, vol. 49, no. 3, pp. 255-261, 2017.

[33] J. X. Kang and A. Leaf, "Prevention of fatal cardiac arrhythmias by polyunsaturated fatty acids," The American Journal of Clinical Nutrition, vol. 71, no. 1, pp. 202S-207S, 2000.

[34] M. T. La Rovere and J. H. Christensen, "The autonomic nervous system and cardiovascular disease: role of n-3 PUFAs," Vascular Pharmacology, vol. 71, pp. 1-10, 2015.

[35] P. D. O. Brien, L. M. Hinder, B. C. Callaghan, and E. L. Feldman, "Neurological consequences of obesity," The Lancet Neurology, vol. 16, no. 6, pp. 465-477, 2017.

[36] Q. Fu and B. D. Levine, Exercise and the Autonomic Nervous System Autonomic Nervous System, vol. 117, pp. 183-184, 2013.

[37] M. C. Freitas, F. L. Ceschini, and B. T. Ramallo, "Resistência à Insulina Associada à Obesidade: efeitos Anti-Inflamatórios do
Exercício Físico," Revista Brasileira de Ciência e Movimento, vol. 22, no. 3, pp. 139-147, 2014.

[38] T. C. B. Lins, L. M. Valente, D. Celestino, S. Filho, and O. Barbosa, "Relação entre a frequência cardíaca de recuperação após teste ergométrico e índice de massa corpórea," Revista Portuguesa de Cardiologia, vol. 34, no. 1, pp. 27-33, 2015.

[39] E. Mehanna, A. Hamik, and R. A. Josephson, "Cardiorespiratory fitness and Atherosclerosis: recent data and future directions," Current Atherosclerosis Reports, vol. 18, no. 5, p. 26, 2016.

[40] S. Aeschbacher, M. Bossard, F. J. Ruperti Repilado et al., "Healthy lifestyle and heart rate variability in young adults," European Journal of Preventive Cardiology, vol. 23, no. 10, pp. 1037-1044, 2016.

[41] C. D. Liao, J. Y. Tsauo, D. J. Hsiao, T. H. Liou, S. W. Huang, and L. F. Lin, "Association of physical capacity with heart rate variability based on a short-duration measurement of resting pulse rate in older adults with obesity," PLoS One, vol. 12, no. 12, pp. 1-16, 2017.

[42] E. M. de S. Carlucci, "Obesidade e sedentarismo: fatores de risco para doença cardiovascular," Comunicação em Ciências da Saúde, vol. 24, no. 4, pp. 375-384, 2013.

[43] C. M. Phillips, "Metabolically healthy obesity: definitions, determinants and clinical implications," Reviews in Endocrine and Metabolic Disorders, vol. 14, no. 3, pp. 219-227, 2013.

[44] P. Wiklund, "The role of physical activity and exercise in obesity and weight management: time for critical appraisal," Journal of Sport and Health Science, vol. 5, no. 2, pp. 151-154, 2016.

[45] S. J. Fonseca-Junior, C. G. A. de Bustamante Sá, P. A. F. Rodrigues, A. J. Oliveira, and J. Fernandes-Filho, "Exercício físico E obesidade mórbida: Uma revisão sistemática," ABCD. Arquivos Brasileiros de Cirurgia Digestiva (São Paulo), vol. 26, no. 1, pp. 67-73, 2013.

[46] A. Fildes, J. Charlton, C. Rudisill, P. Littlejohns, A. T. Prevost, and M. C. Gulliford, "Probability of an obese person attaining normal body weight: cohort study using electronic health records," American Journal of Public Health, vol. 105, no. 9, pp. e54-e59, 2015. 